\title{
Preface to the Special Issue: Nonlinear systems theory and applications in engineering, control and life sciences
}

\author{
Jan Awrejcewicz • Björn Birnir • \\ Miguel A. F. Sanjuán
}

Published online: 6 July 2019

(C) Springer Nature B.V. 2019

We are proud to present this Special Issue of Nonlinear Dynamics, which is devoted to the 14th International Conference "Dynamical Systems - Theory and Applications" (DSTA 2017) that took place December 11-14, 2017, in Łódź, Poland.

The DSTA conference series organized since 1992 by the Department of Automation, Biomechanics and Mechatronics of Lodz University of Technology is held every 2 years. It gathers researchers working in the broad field of nonlinear dynamics. DSTA 2017 was attended by 250 scholars from 35 countries, who gave 140 speeches selected from 370 submitted abstracts.

Some remarkable contributions were recommended for inclusion in the present Special Issue. To ensure the highest quality of the content, all manuscripts were significantly extended and underwent a rigorous peerreview process. As a result, 12 contributions related to nonlinear systems theory and applications in engineering, control and life sciences were accepted to be published in the Special Issue. A brief summary of these contributions is provided next.

\footnotetext{
J. Awrejcewicz ( $\square)$

Department of Automation, Biomechanics and

Mechatronics, Lodz University of Technology, Lodz,

Poland

e-mail: jan.awrejcewicz@p.lodz.pl

B. Birnir

Center for Complex and Nonlinear Science, University of California, Santa Barbara, USA
}

M. A. F. Sanjuán

Nonlinear Dynamics, Chaos and Complex Systems Group, Rey Juan Carlos University, Madrid, Spain
Antali and Stepan analyzed the nonsmooth dynamics of two contacting rigid bodies in the presence of dry friction. Aside from taking into account slipping and the Coulomb friction model, they applied the new theory of extended Filippov systems to analyze the dynamics of a rigid body moving on a fixed rigid plane to explore the possible transitions between the slipping and rolling behavior. Their research focuses on finding the so-called directions of the slipping equations at the discontinuity, and the employed methods used are also demonstrated in the context of an example.

Amabili studied the topic of damping which is observed to increase with the vibration amplitude in both macro- and nanoscale structures. He derived nonlinear damping from viscoelasticity by using a singledegree-of-freedom model obtained from the standard linear solid material including the geometric nonlinearity. To obtain a solution, a third-order harmonic balance method was used. Experimental and numerical results are compared for forced vibration responses measured for two different continuous structural elements: a freeedge plate and a shallow shell.

The paper by Awrejcewicz et al. focuses on the dynamics of the micromechanical gyroscope designed for measuring one component of the angular velocity. It considers both nonlinear effects of the geometrical nature and the nonlinear characteristics of the torsional joints. A second-order Lagrange equation is used to derive the mathematical model of the system. An analytical form of the approximate solution was obtained 
by the method of multiple scales for nonresonant vibration as well as for the case of the simultaneous presence of the primary and internal resonances.

Breunung et al. conducted the stability analysis of linearized mechanical systems with an arbitrary number of degrees of freedom, time-periodic damping and stiffness matrices. They used a novel approach capable of accounting for the interference between the averaged eigenvalues. To verify the validity of the employed approach, they revisited two example systems from rotor dynamics.

In their paper, Dzyubak et al. focused on new designs and optimization of the cancer treatment. For this purpose, they studied the nonlinear multiscale diffusion cancer-invasion model that describes the interactions of the tumor cells, matrix-degradative enzymes and oxygen. They obtained the regions of parameters causing the carcinogenesis of the studied biological system.

The work of Fidlin and Aramendiz Fuentes concerns the dynamics of a system supplemented with a tuned lockup mass damper. The authors applied a special analytical approach based on rescaling of variables to obtain analytical predictions of the steady-state amplitudes for both the main system and the damper. The obtained results show that the damper cannot provide perfect vibration suppression at any tuned frequency, despite the ability to limit the amplitudes at resonance for a wide range of parameters.

Gidlewski et al. verified the assumption that a predominating part of the energy lost at vehicle collisions is consumed by permanent deformation of the vehicle components, and therefore, other negligible parts of the energy dissipated can be ignored. The authors considered the front-to-side collision of passenger cars to determine the important components of the energy balance dissipated during the collision. They developed a model of the dynamics of a motor collision based on experimental results. The obtained results can contribute to the reconstruction of a front-to-side collision of motor vehicles.

Hafstein and Valfells focused on the Lyapunov function for the equilibrium of a dynamical system. They proposed, improved and analyzed a method that is based on a converse theorem for the Lyapunov stability theory, and then compared it with other methods from the literature. The approach they proposed is beneficial in comparison with other existing methods as it is of lower complexity, its workload is perfectly parallel, and its free parameters allow it to be adapted to the problem at hand.

Martowicz et al. developed a peridynamic model for a solid body made of shape memory alloys (SMA). The employed SMA model describes the solid phase transitions giving rise to pseudoelasticity. Numerical simulations are conducted to investigate mechanical energy dissipation. The model is validated using experimental results, and a finite element approach. Possible applications of the model are also discussed.

In their study, Yang et al. integrated a nonlinear energy sink (NES) and a negative stiffness element for pursuing enhanced, passive, and adaptive vibration suppression in a pipe conveying variable flow velocity. The enhanced NES can simultaneously account for restoring forces associated with negative linear and cubic nonlinear spring effects obtained by two linear springs with a special configuration and preloaded deformation. The authors derived and simulated the governing equation of the NES-pipe system to investigate vibration mitigation effectiveness.

Zelei et al. studied the topic of human and robotic legged locomotion. They focused on the effect of kinematic parameters and foot placement techniques on the ground-foot impact intensity. Their work is based on a multibody dynamic model of a segmented leg, which possesses some fundamental characteristics of locomotion systems, such as kinetic energy absorption due to partially or fully inelastic ground-foot collision or different control strategies for the stance and swing phases. They analytically obtained a quantitative measure of the foot collision intensity. Moreover, they investigated how the stability of the periodic motion depends on the control gains.

We wish to acknowledge all contributors for the efforts put forth in their high-quality scientific papers. Furthermore, we would like to express our greatest gratitude to all reviewers involved in the peer-review process. Finally, we thank the Editor-in-Chief of Nonlinear Dynamics, Professor Walter Lacarbonara, for supporting the publication of this Special Issue.

We believe that the readers of Nonlinear Dynamics will find the content of this Special Issue interesting and inspiring for their own research in the field of dynamical systems.

Publisher's Note Springer Nature remains neutral with regard to jurisdictional claims in published maps and institutional affiliations. 